\title{
Suggested Procedures for Operationally Integrating Meteorological Satellite and Conventional Data for Extratropical Regions ${ }^{1}$
}

\author{
PaOL E. Sherr \\ ARACON Geophysics Company, Concord, Mass. \\ (Manuscript received 19 March 1965, in revised form 12 November 1965)
}

\begin{abstract}
The integration of satellite and conventional data can lead to an improved meteorological analysis and interpretation. Procedures for such a data integration are suggested. The advantages that can be gained from this data integration and time-tested techniques, such as continuity, are demonstrated by case examples both for areas of sparse and relatively abundant conventional data. It is shown that, over data sparse areas, the techniques provide a means of obtaining at least a qualitative analysis where none could (or should) be made otherwise. Even for areas of relative data abundance, the techniques can provide improved analyses at the synoptic as well as smaller scales.
\end{abstract}

\section{Introduction}

Most of the published literature on satellite meteorology emphasizes what the TV camera observes, and the interpretations that have been made from these observations by research workers. This paper will deal more specifically with suggested operational procedures for applying these data at forecast stations. These procedures will become even more useful with the more routine observations to be made by the TIROS Operational Satellites (TOS) now scheduled to begin in early 1966. The TOS system should provide data every day on a global basis and at approximately the same local time. Present satellites provide a new source of important data for synoptic analysis and forecasting on a nearly global basis (Widger, 1966).

Experience has shown (Miller, 1963; Widger, Sherr and Rogers, 1964; Oliver, 1962) that reasonable estimates of large scale trough and ridge patterns, for at least the middle level in the atmosphere, can be deduced in most cases from satellite data, especially when climatology and analog techniques are judiciously applied. However, it is important to note that the use of satellite observations should complement, not replace, analyses based on available conventional data. The operational value of satellite data often depends upon the relative adequacy of the conventional data otherwise available to the analyst and, hence, varies widely with geographical location. Even for areas of relatively abundant data, satellite data can often improve synoptic analyses based principally on conventional data. In data sparse areas, satellite data, coupled

\footnotetext{
1 The techniques presented in this paper resulted from research conducted by ARACON Geophysics Company, chiefly under sponsorship of the U. S. Air Force Cambridge Research Laboratories. They were originally published in "Practical Interpretation of Meteorological Satellite Data," Final Report, under Contract No. AF 19(628)-2471, ARACON Geophysics Company.
}

with meager conventional observations, will generally lead to a far better synoptic analysis than would otherwise be possible. In either case, satellite and conventional data should be integrated in a manner convenient to the forecaster and one that allows the maximum value to be gleaned from the combined data sources. It is worth recalling that a forecast can seldom be better than the analysis on which it is based.

\section{Suggested techniques for integration of conven- tional and satellite data}

The use that a forecaster makes of satellite data depends on many criteria, but the foremost is usually the relative amount or quality of conventional data available to him. The following suggested techniques were developed for optimum utilization of the data and are designed so they can be accomplished in the field. However, these techniques can be even better accomplished at weather centrals since increased manpower and possibly actual satellite pictures can be applied there. Some of these techniques are already in use at the National Weather Satellite Center.

Neph mosaics. Present practices allow only limited. facsimile time for the transmission of nephs, and this and the nature of TIROS orbits have resulted in nephs being received at somewhat irregular intervals. A definite deficiency in the present system is that of providing the nephanalyses to the forecaster as limited pieces of satellite data for only small portions of the earth at a given time. Use of the data in this form is analogous to the exclusive use sectional maps without the benefit of larger area analyses.

Accordingly, available adjacent or nearly adjacent nephs should be mosaiced together. The nephs can be pasted or stapled to an appropriate base map, as in the composite nephanalysis shown in Fig. 1. 

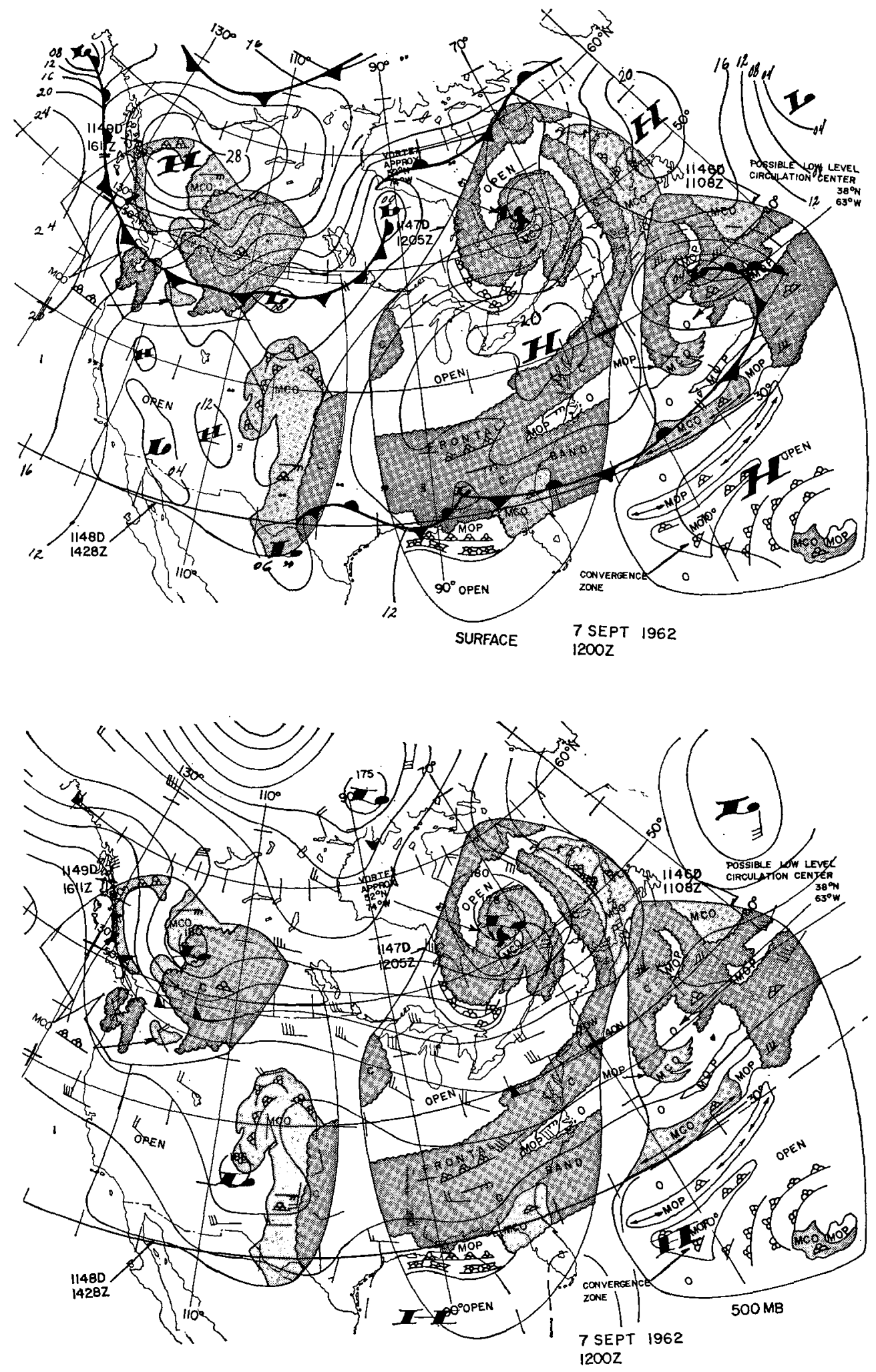

FIG. 1. Neph mosaic with superpositioned conventional surface and 500-mb analysis for 1200 GMT, 7 September 1962 . 
Experience has shown that all nephs within $\pm 6 \mathrm{hr}$ of a standard analysis time can effectively be so composited, since the large scale features of a cloud system retain their representative patterns for several hours (Brodrick, 1964). The neph mosaic allows the spatial continuity of cloud patterns to be easily seen.

In general, a new twelve hour composite should be started at neph times which are approximately six hours after each standard analysis time. Where these specific intervals would separate the data near the area of prime interest, other time intervals may be more appropriate. As each new orbit is received, it should be added to the composite. Where overlap occurs, the neph closest to the standard analysis time should be given preference unless the other orbit provides a better depiction of the cloud patterns, or the latest orbit shows significant subsequent developments.

Shading of significant patterns. Pattern recognition is made easier if "Covered" (C) areas are shaded in color $^{2}$ (for example, blue) and "Mostly Covered" areas (MCO) are also shaded in the same color but in a lighter shade. This shading, in addition to any stippling ${ }^{3}$ that appears on the nephs, makes the recognition of significant synoptic features easier since it maintains the continuity of cloud patterns both within a single neph and between adjacent nephs in the mosaic.

Superposition of conventional and satellite data. Experience has shown the following suggested procedures will aid operational utilization of the satellite data. There are indications that future transmissions from weather centrals to field forecast stations are likely to resemble this format.

1) Oceans and other areas of sparse conventional data. The greatest need for cloud data over oceans is usually at the synoptic scale, and it is suggested that the available nephanalyses be integrated with a concurrent analysis. Examples are shown in Figs. 1 and 3-8. General experience indicates that usually extratropical cloud patterns are most usefully correlated with the 500-mb chart. Other charts, such as a surface map, can also be used; but the integration of the neph mosaic and the $500-\mathrm{mb}$ chart nearest to the neph time is usually found to be the preferable procedure. In a critical operational situation, however, a check with the surface analysis may be useful.

An important factor in simplifying the integration task is that both types of data are on compatible map projections. It is, therefore, relatively easy to trace the major features of the conventional analysis onto the neph mosaic.

For a data sparse area an up-to-date and reasonably reliable prognostic chart can often assist an analysis

${ }^{2}$ For this publication, only shades of gray were permissible. The use of color is far-more effective and is suggested to users of these techniques.

${ }^{3}$ Stippling on the transmitted neph defines areas considered by the analyst preparing the neph as of the greatest synoptic significance. It should be considered a guide and not as an irrevocable decision. which is to be based primarily on the satellite observations. The prognostic chart will indicate the types of synoptic features expected to exist over the area, their location, and (unless unexpected developments are indicated by the satellite cloud pattern) the pressures and contour heights at the centers of lows and highs. The satellite data can then be used to improve ard modify the analysis by showing the actual locaticns of the features and by identifying developments not anticipated in preparing the prog chart. (See Figs. 9-12.)

2) Land areas with adequate conventional data. Over continental areas where adequate conventional data exists, there may be some question whether routinely combining the large scale cloud patterns and operational synoptic charts is a sufficiently useful standard procedure to justify the time and effort required. Generally, this tradeoff will hinge on the operational and weather situations.

For stations where adequate nephs are available, and especially for $\mathrm{AP}^{\prime}{ }^{4}$ locations, more detail is afforded by the pictures than is presently possible in the nepts. Thus cloud patterns on a scale approaching mesoscale can aid the forecaster. Such significant weather producers as squall lines or large cumulus buildups (Fig. 2) can often be seen in the satellite pictures and be more precisely located. Overlapping passes of the satellite may give some indication of the development of such features.

There will be many times when it is important to know, either for forecasting or flight briefing, precisely where the boundary of a synoptic scale cloud system lies. At other times, it may be of practical significance to know something about the organization of cloud elements within a particular cloud mass. In either case, the cloud data might be appropriately transposed to the Weather Depiction Chart. In both cases, significant details of the cloud systems are usually not revealed by conventional data.

When satellite data are used at this scale of precision, consideration should, of course, be given to possible errors in the determination of geographic position. Although the relative positions of cloud masses are well depicted in the satellite data, their precise geographical positions (at the accuracy required for mesoscale application) may be questionable unless adjacent clear areas reveal landmarks which can serve as a check on the position accuracy.

The data integration procedures suggested above are applicable to APT data, even though rectification and scale considerations require "eyeball" transposition of the cloud data to the conventional analysis, or vice versa. Specific procedures will necessarily depend on local station procedures. At stations where larger scale sectional charts are analyzed, the appropriate cloud details are probably best transposed to this chart.

\footnotetext{
${ }^{4}$ A utomatic Picture Transmission which was successful on both TIROS VIII and Nimbus I (Stampfl and Stroud, 1963).
} 
What information to transpose and how best to transpose it will depend on the nature of the meteorological situation as well as on the nature of the forecast problem. Hanson (1963) has suggested transposing conventional data onto the picture when there are significant details in the photo which can easily be retained in a symbolic nephanalysis. It is desirable to coordinate APT data with a sectional map for approximately the same time.

\section{Demonstration of procedures}

Nephanalyses and pictures are presented for cases in September 1962 and April 1963 to demonstrate the improved synoptic analyses that result from combination of the satellite and conventional data.

A. An autumn case. Nephs covering areas of the United States and the adjacent Atlantic Ocean have been prepared as mosaics for the period 7-10 September 1962. The top of the nephanalyses in this paper shows the surface analysis superposed over the neph mosaic while the lower portion shows the corresponding $500-\mathrm{mb}$ analysis superposed over the same mosaic. Fig. 2 shows a portion of orbit $1147 \mathrm{D}$ upon which a part of the nephanalysis shown in Fig. 1 was based.

Only one major pattern will be discussed, although other features can easily be recognized and will be briefly listed at the end of this section.

This pattern is associated with the $500-\mathrm{mb}$ trough over the mountain states, which developed into a major storm.

An upper level development over the Mountain States. At 1200 GMT 7 September (Fig. 1), two separate systems appear on the map: (1) a small closed low centered over northern New Mexico and (2) the trough over British Columbia which shows a closed isoline although only limited organization is apparent in the nephanalysis. During the period from 1200 GMT 7 September, to and including 0000 GMT 9 September, this second system was analyzed by NMC as having a single closed contour but it would be difficult to either confirm or deny from conventional data the validity of this closed contour. Prior to 9 September the behavior and synoptic significance was more that of a trough than a closed low in the middle troposphere. The cloud patterns more closely resembled patterns usually associated with an open trough than those representative of a closed $500-\mathrm{mb}$ system (Widger, 1964). The lack of cloud patterns characteristic of a closed low may have indicated a minimal closing off of the system during this relatively extended period.

Over New Mexico, the conventional 500-mb analysis indicates a short wave is passing around the southeast side of the closed system. The cloud pattern again does not indicate a closed system but it consists of middle or low clouds as shown by the cumuliform symbols on the neph. It appears from the combined neph mosaic and surface chart (Fig. 1) that this cloudiness is associated with the middle level system and not with any well organized surface or low level system. A rather wide area of precipitation is occurring with this southern system which might not have been expected using conventional contour or pressure data alone.

Fig. 3 shows the patterns for 0000 GMT 8 September. A large covered area, presumably extending north of the area of observation, now correlates well with the $500-\mathrm{mb}$ center over the Canadian-United States border

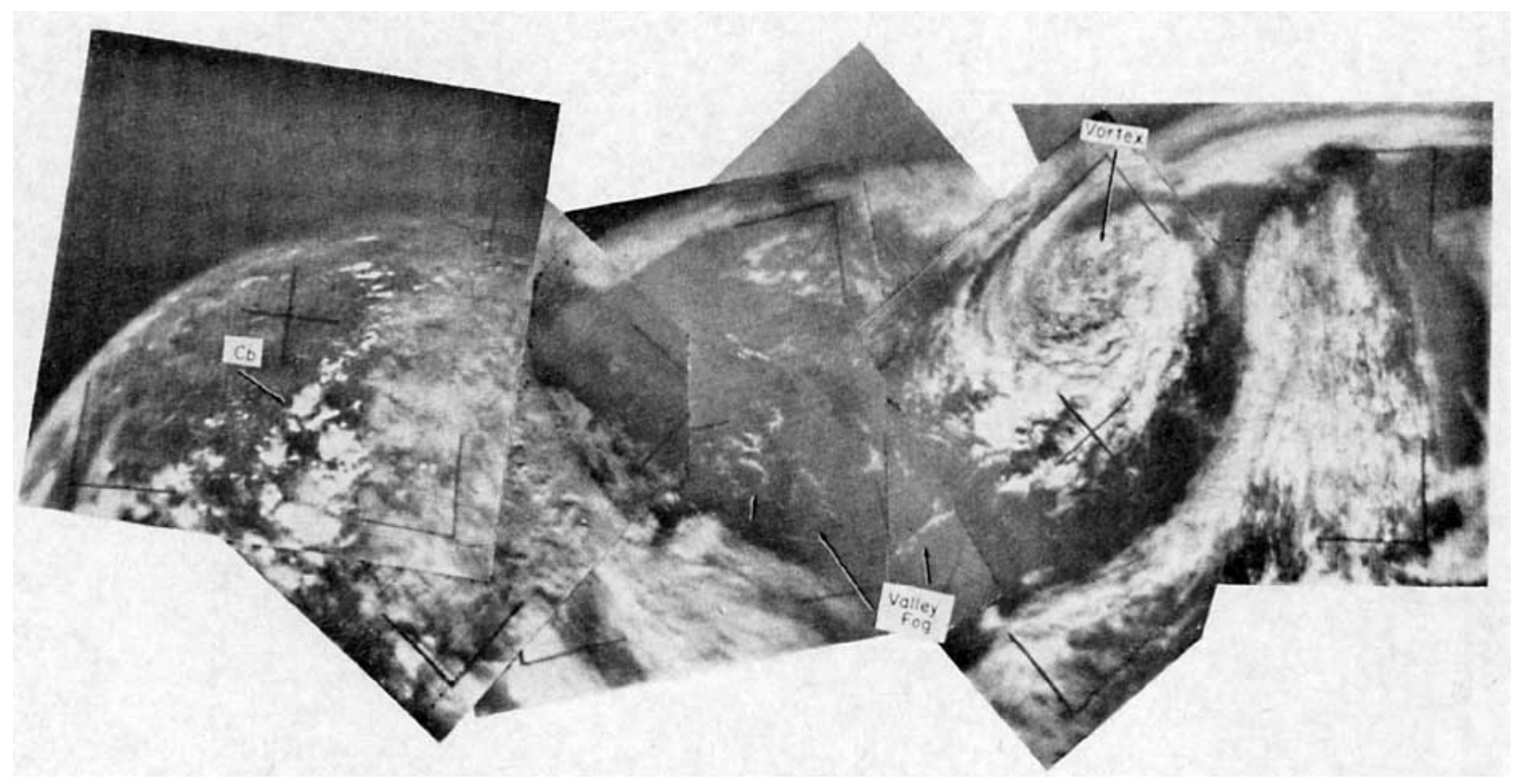

FIG. 2. Mosaic of TIROS V, orbit 1147D, 7 September 1962, 1250 GMT. (Notable features are a well developed vortex, clear areas, valley fog patterns, and line of $\mathrm{Cb}$.) 

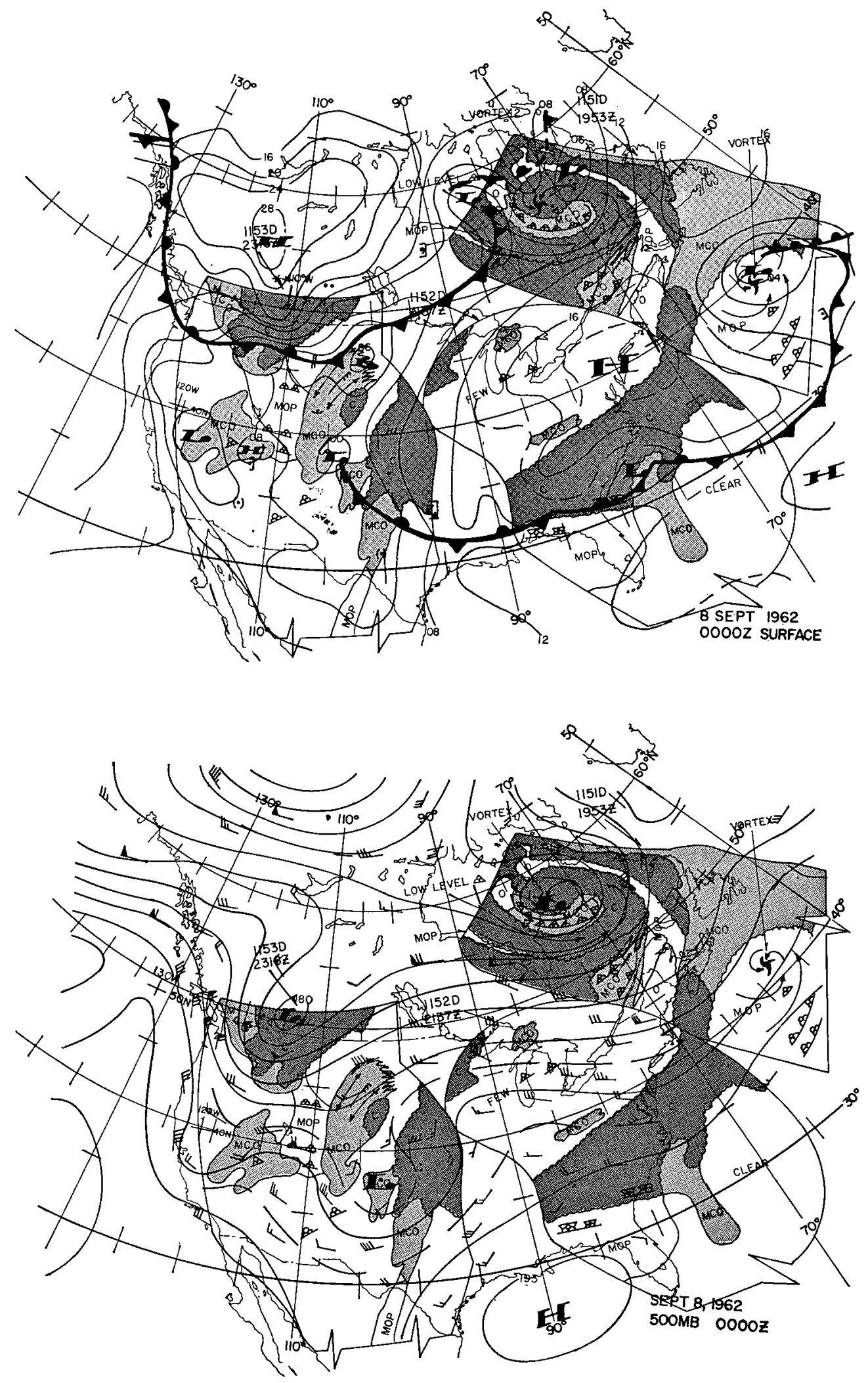

Fig. 3. Neph mosaic and conventional analysis for 0000 GMT, 8 September 1962. 

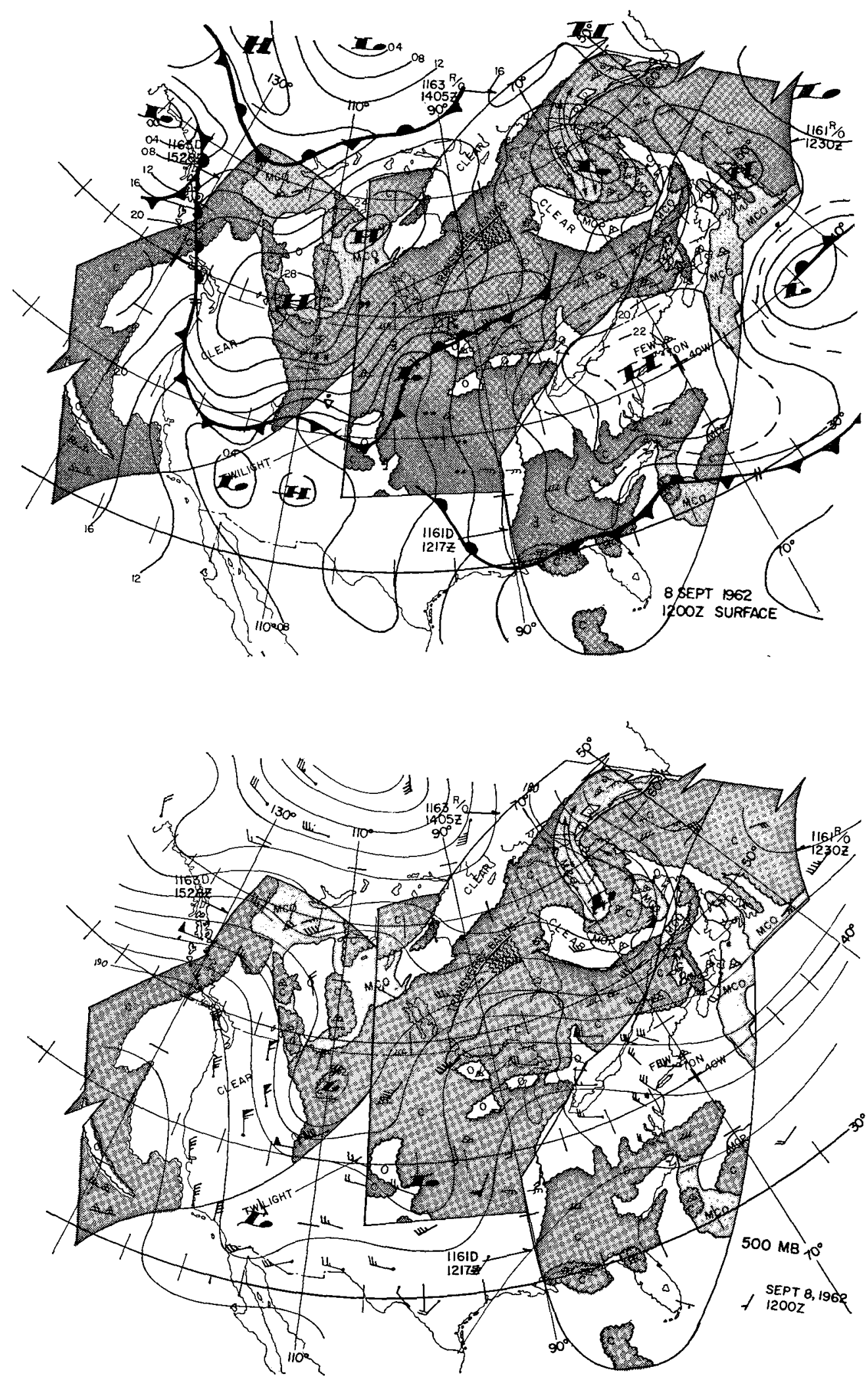

FIG. 4. Neph mosaic and conventional analysis for 1200 GMT, 8 September 1962. 
near $112 \mathrm{~W}$. Snow and rain have broken out. However, the cloud pattern does not fit well with the frontal analysis. In a data sparse area, this cloudiness might not have been expected and the resultant precipitation might not have been forecast without the satellite data.

The closed 500-mb contour pattern, formerly over New Mexico, now shows as an open trough over northern Texas and Oklahoma. Again the cloudiness correlates well with the $500-\mathrm{mb}$ pattern but not very well with the surface pattern. Over a data sparse area, this "blob" of cloud might aid in positioning an upper level trough. A trough which at 1200 GMT 7 September was just moving onto the Oregon coast can now be seen both in the contour pattern and in the neph over western Nevada.

By 1200 GMT 8 September (Fig. 4), the southernmost system is still over northern Texas and Oklahoma, and appears as a short wave moving around the major northern center which is now near $47 \mathrm{~N}, 110 \mathrm{~W}$. A second short wave, formerly over western Nevada, is now over southwestern Arizona but the neph does not permit a useful integrated analysis of this area. The short wave over Oklahoma now has a heavy cloud cover associated with it but no closed circulation is apparent. A frontal wave shows signs of development immediately downwind of the upper short wave. Moderate precipitation continues to be associated with this short wave. The associated cloudy area now extends eastward into the cloudiness associated with the major trough over eastern Canada. The clouds still do not reveal the pattern usually representative of a closed circulation at $500 \mathrm{mb}$ and the trough along $110 \mathrm{~W}$ is at best barely closed.

Another heavy cloud system moving into western Canada and the United States can be seen near 130W. This cloudiness suggests a wave with a greater amplitude than that in the conventional analysis.

Fig. 5 for 0000 GMT 9 September shows the closed $500-\mathrm{mb}$ center to be near $45 \mathrm{~N}, 108 \mathrm{~W}$. The cloud pattern in the neph is no more than hook shaped, which is usually characteristic of an open trough rather than a closed system (Fig. 5, Widger, 1964; Figs. 6-8, Widger et al., 1964). A band now extends south and southwestward along the associated trough and the short wave that was over southwest Arizona 12 hours earlier. This band does not fit the surface analysis; this suggests that either the frontal analysis is incorrect or that the frontal analysis was not a good indicator of the "weather" in this situation.

The heavy cloud "blob" associated with the short wave moving around the eastern side of the major trough over Wisconsin, Illinois, and Iowa is easily recognized and is the producer of substantial precipitation. This short wave is moving rapidly and the difference in neph time (2240 GMT) and analysis time (0000 GMT) probably accounts for the cloudiness which appears to be in the trough line. The cloudiness to the west of the trough line is broken; since it is near the horizon in the picture, it might be slightly misplaced in the neph (errors in position of up to 3 degrees are possible for features near the horizon). The ridge line downwind from this short wave is clearly depicted by the mostly open area between the cloud masses associated with this trough and with the $500-\mathrm{mb}$ system farther to the east. Over data sparse areas, an analyst could use such an indication of breaks to place a ridge line.

The maps for 1200 GMT 9 September (Fig. 6) continue to show the good correlation of the cloud pattern to the 500-mb data. The hook shaped cloud mass over the Dakotas, with its trailing band downwind from the trough line, is not quite as evident as 12 hours previously. This is probably a function of the lack of neph coverage and illustrates how continuity aids in the recognition of the pattern. This hook shaped pattern is associated with the 500-mb flow and not with the surface flow.

The cloud pattern associated with the short wave (which is over the Greak Lakes, well up the eastern side of the major trough) has a more organized pattern and is now indicated to be a "possible vortex." This possible vortex probably marks the position of the surface system. A cloud mass showing some semblance of a band seems to be trailing south and then southwestward from this area just downwind of the trough. This band is more closely associated with the position of the front, as drawn on the surface analysis, than the remaining mass of clouds still farther west. The low level center is complex in nature but centered approximately under the upper level short wave trough. Most of the precipitation of the system is associated with this short wave system. The cloudiness in advance of this short wave pattern extends well into the ridge at this time and seems to join the cloud area associated with the system to the northeast.

In Fig. 7, 0000 GMT 10 September, the winds north of the center now leave little doubt that the principle center at $500 \mathrm{mb}$ has a closed circulation. Some increase in the cloud organization is apparent but the pattern still does not resemble the pattern expected for a closed 500-mb system (Widger, 1964). A cloud band still extends to the south just downwind of the longer wave trough. The short wave trough has now moved into the area of the long wave ridge causing a more zonal flow pattern. The covered area near $48 \mathrm{~N}, 75 \mathrm{~W}$ is probably associated with the remnants of this short wave trough, while the breaks in the cloud pattern near Lake Huron probably indicate the position of a weak ridge. Widespread precipitation has now broken out and covers a large part of the central United States. The westward boundary of this precipitation could be better deduced from the movement of the rear boundary of the cloud mass than from frontal positions.

Fig. 8 shows the continued development of the storm with the $500-\mathrm{mb}$ and vortex centers at $45 \mathrm{~N}, 90 \mathrm{~W}$. 

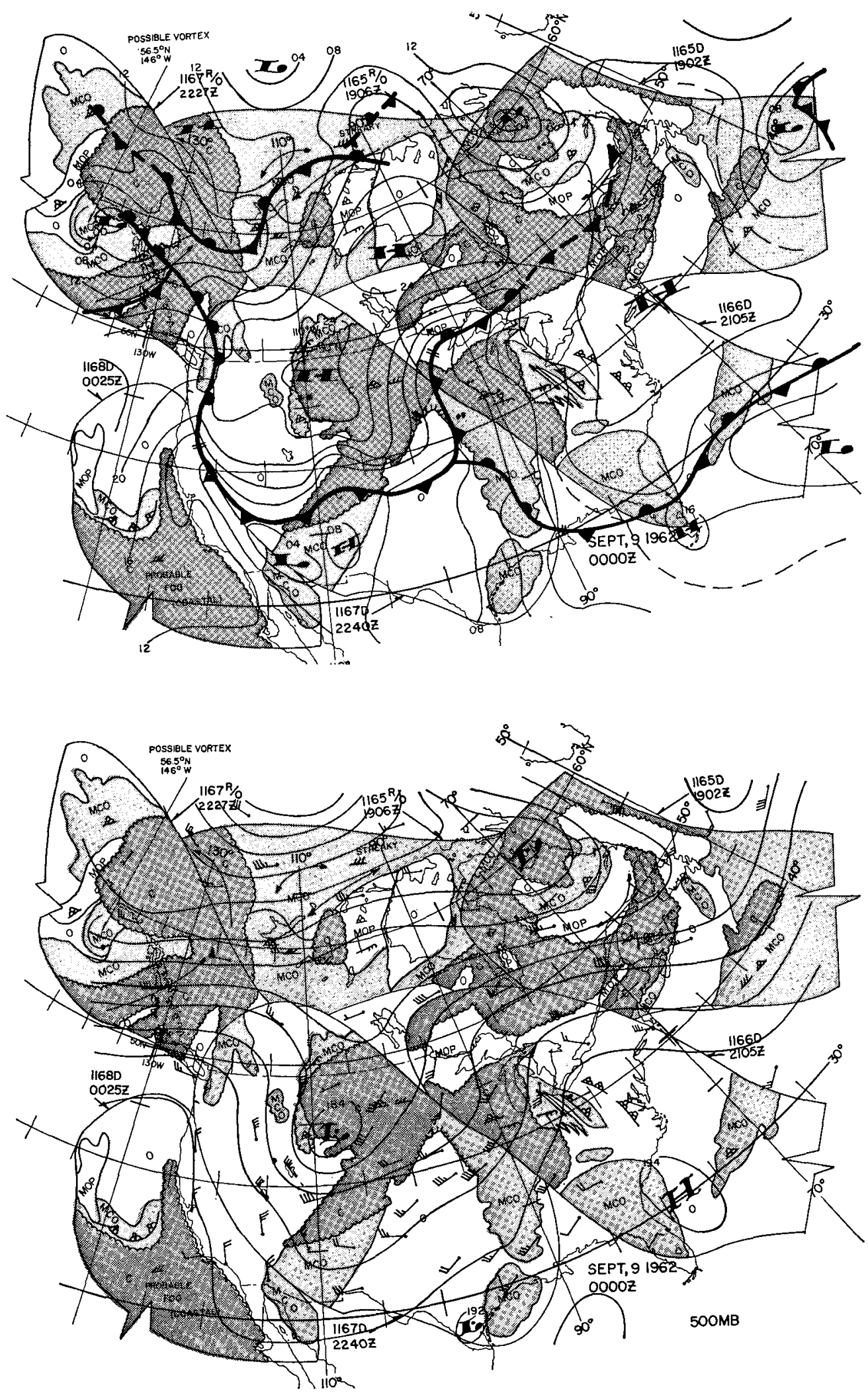

FIG. 5. Neph mosaic and conventional analysis for 0000 GMT, 9 September 1962. 

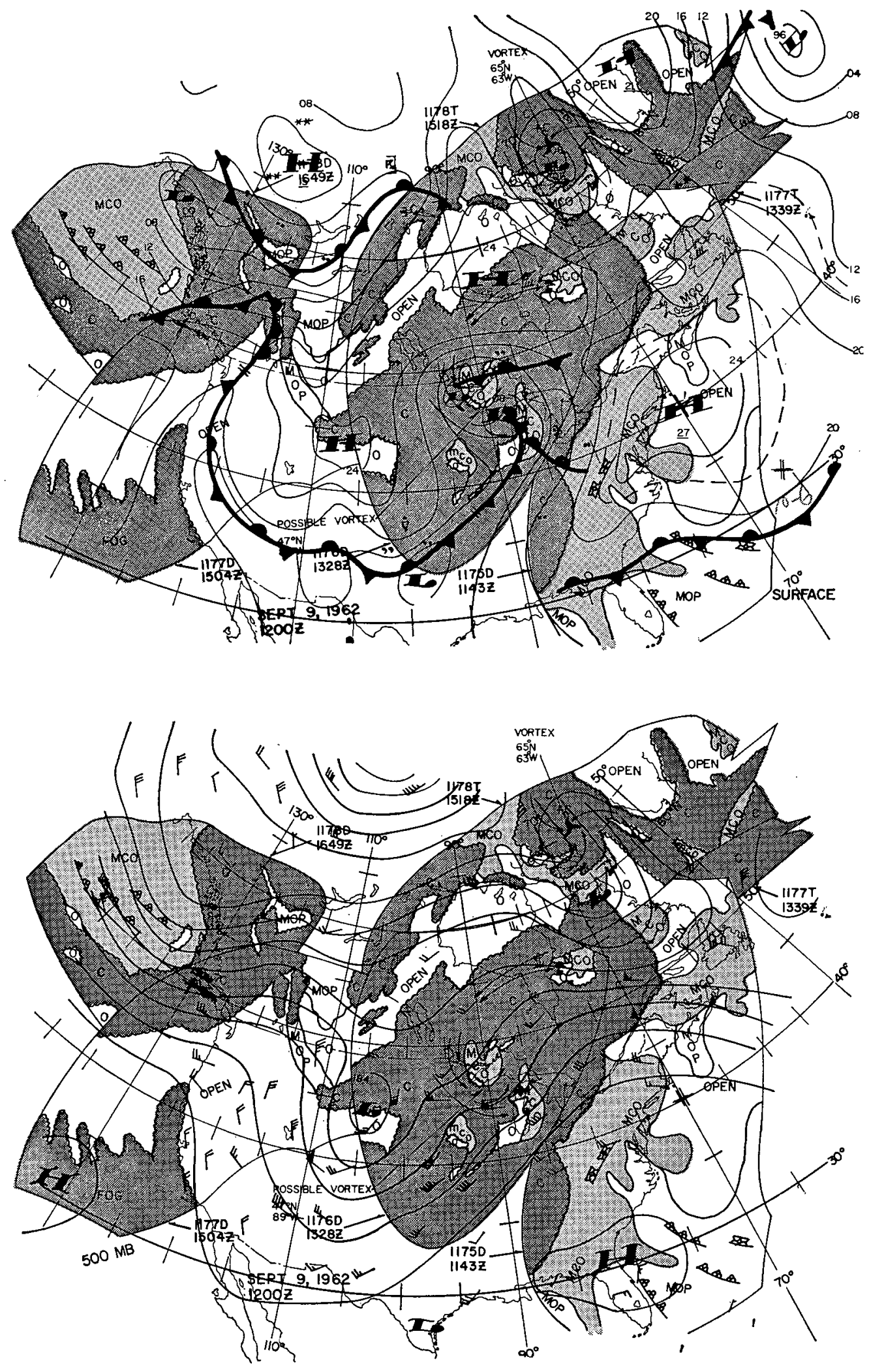

FiG. 6. Neph mosaic and conventional analysis for 1200 GMT, 9 September 1962. 

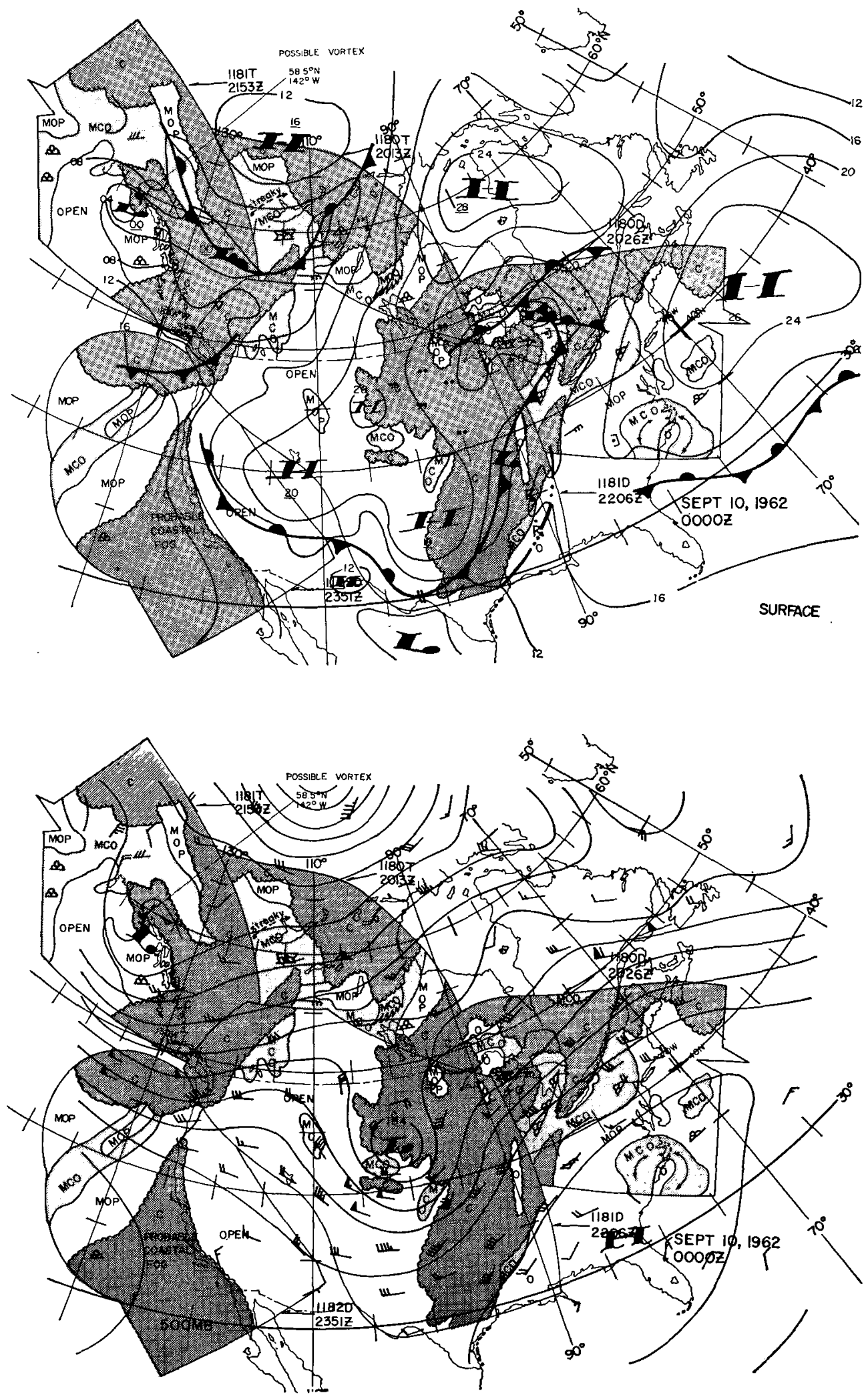

Fig. 7. Neph mosaic and conventional analysis for 0000 GMT, 10 September 1962. 

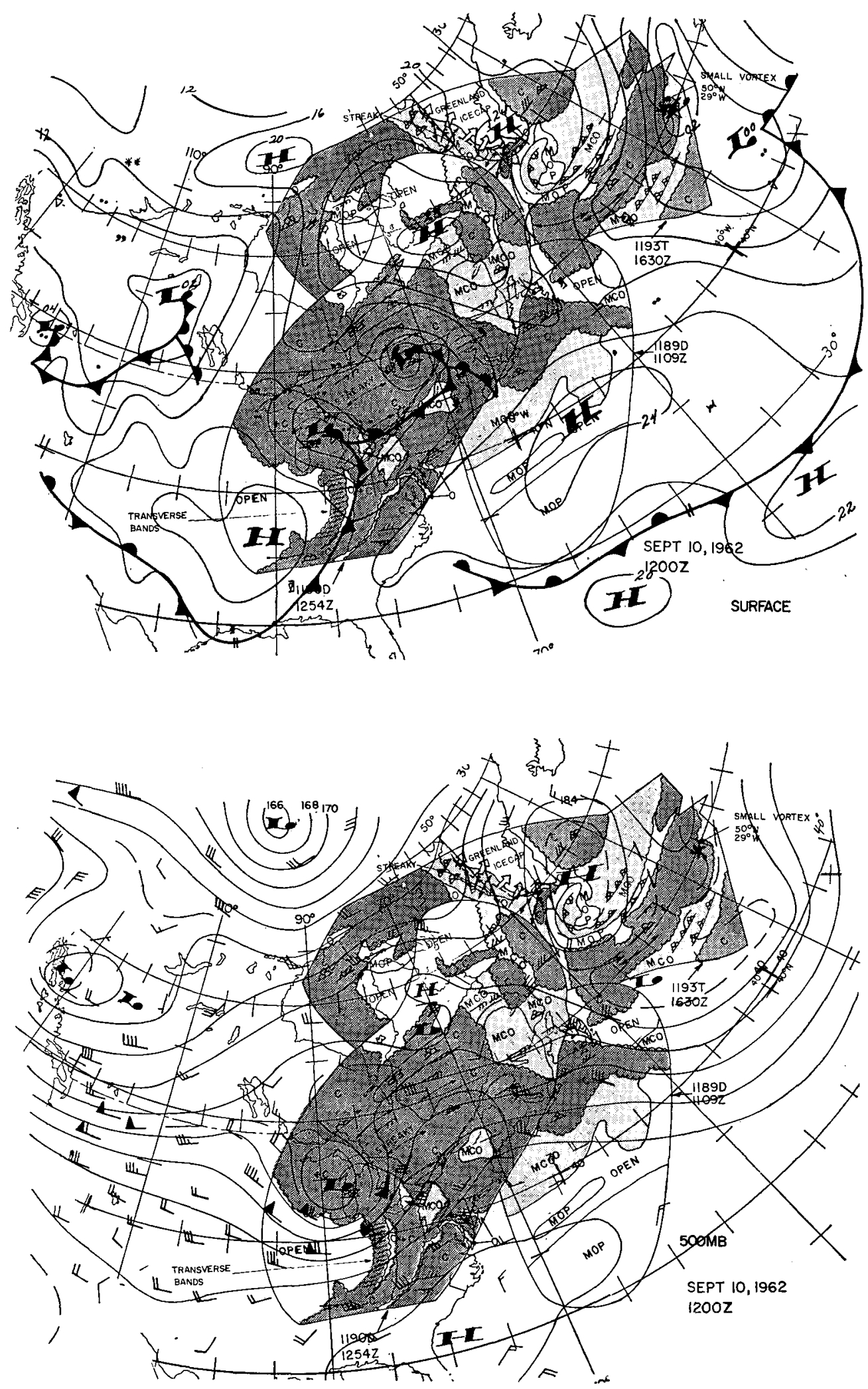

Fı. 8. Neph mosaic and conventional analysis for $1200 \mathrm{GMT}, 10$ September 1962 . 

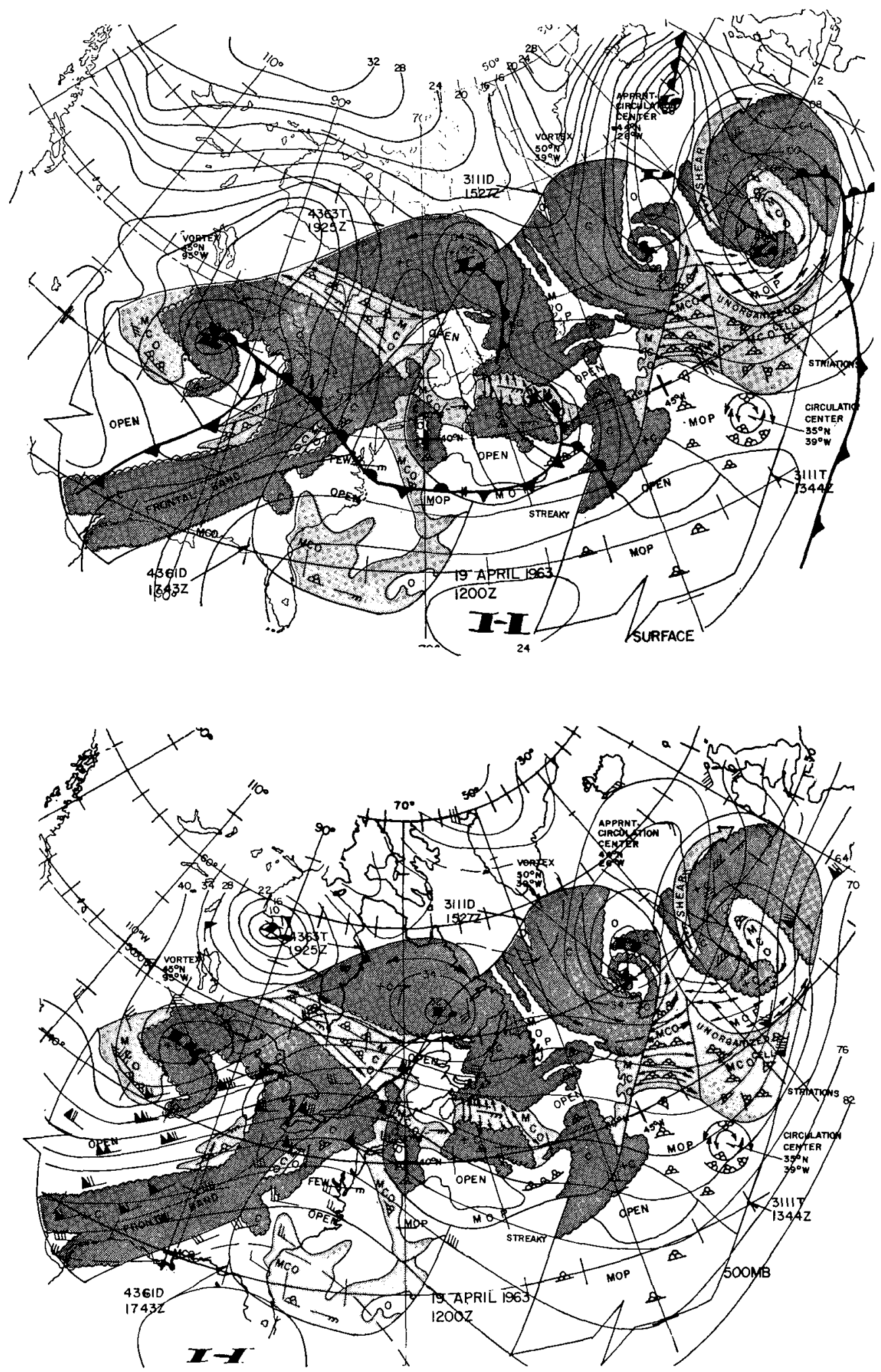

FIG. 9. Neph mosaic and conventional analysis for 1200 GMT, 19 April 1963. 


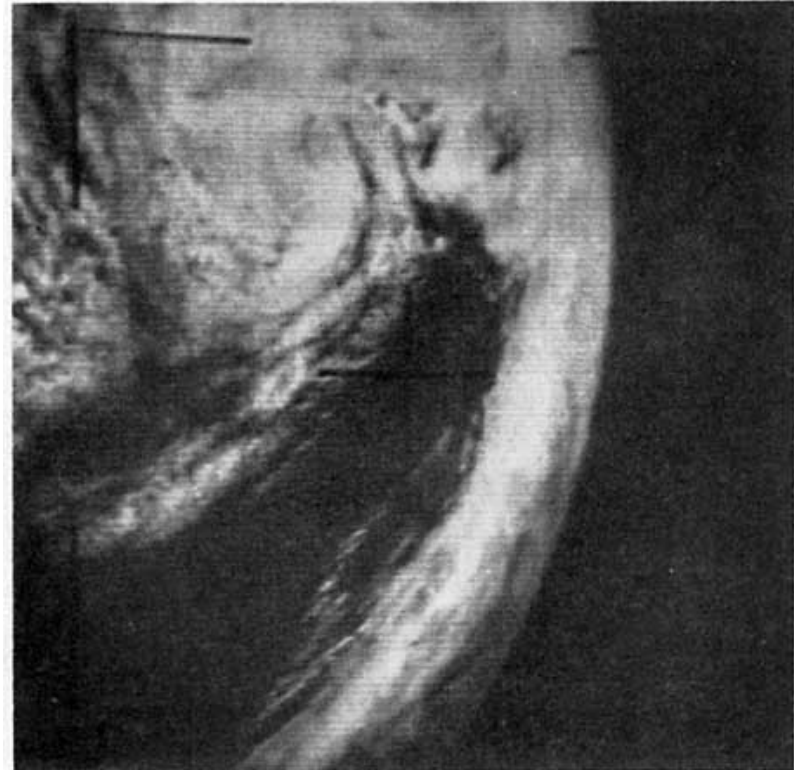

FIG. 10. Picture from orbit 4363 R/O 4362 TIROS V, 1925 GMT, 19 April 1963.

The precipitation is confined mostly to the north and northeast of the center in the areas depicted as heavy covered and covered. Some evidence of the short wave trough and its associated vorticity maximum can still be seen in the cloud pattern over the Gulf of the St. Lawrence near $47 \mathrm{~N}, 60 \mathrm{~W}$. Precipitation is still occurring in this area well in advance of the surface frontal system and indeed in the surface ridge. By 1200 GMT the next day (11 September 1962) the vortex was centered at $48 \mathrm{~N}, 80 \mathrm{~W}$ and looked remarkably like a vortex which moved through the area four days earlier (shown in Fig. 2).

Other significant palterns. Many other significant patterns are recognizable in this set of neph mosaics; some of the more obvious are:

1) The major cyclone over Eastern Canada (Figs. 1 and 2) developed from a frontal wave associated with middle tropospheric cyclonic vorticity advection (Boucher et al., 1963). This storm weakens and reintensifies and weakens again as it moved eastward during the period 7-10 September. Clues regarding the reintensification that can be obtained from the combined analysis of the satellite and conventional data are discussed in Widger et al. (1964).

2) The extensive area of stratiform and cumuliform cloudiness off the west coast.

3) The cloudiness moving onto the west coast of Canada on 8 September at 1200 GMT. The comma shaped pattern on 9 September suggests an approaching upper trough and possibly an associated low level occluding storm. By 1200 GMT 9 September, the trough is evident and the typical banded cumuliform cloudiness in the cold air behind the upper level trough line is clearly depicted.
4) In Fig. 6 the short wave trough along $105 \mathrm{~W}$, near $56-60 \mathrm{~N}$, could be suspected from the cloud pattern, even in the absence of radiosonde data.

5) The lines of cumulonimbus along the Gulf Coast on 7 and 8 September are easily related to thunderstorm activity for this area and illustrate the identification of mesoscale convective patterns.

B. A spring case. In this case, for 19-21 April 1963, coverage on the first day is over the central and eastern United States, Canada, and into the western Atlantic. On 20 and 21 April, the synoptic systems moved over the data sparse Atlantic where the integration of the data materially aided in constructing a better analysis. Three situations are discussed in some detail.

Vorlex pattern over Minnesota. The composite nephanalysis for 19 April (Fig. 9) shows a cloud vortex over Minnesota. From the TIROS picture (Fig. 10) this storm appears to be at the "beginning of occlusion" stage (Widger, 1964). Except for the orientation of the frontal band, this case, including the upper level trough position and flow pattern, fits Widger's example. The neph for orbit 4363 is closer to the 0000 GMT analysis time but it has been included with the other orbits (which are closer to $1200 \mathrm{GMT}$ ) for the purposes of continuity of coverage over the area under discussion.

The vortex over Minnesota (Fig. 9) is associated with a low pressure system with a vertical axis. The compositing of the nephs allows the eastern boundary of this system and the frontal band to be clearly depicted. This band suggests that the frontal system at about 1800 GMT (near the neph time) should be nearly as far east as it was analyzed to be on the 0000 GMT surface analysis (not shown). A forecaster can use such positions of fronts and upper level troughs relative to the cloud pattern [as shown in Widger (1964)] to aid in both surface and middle tropospheric analyses.

When a frontal band extends well equatorward of a vortex center, an abrupt ending or a splitting of the band usually indicates the position of the upper level trough line (Oliver, 1962). In Fig. 9, the frontal band is split near Houston. Considering the time difference between the neph (1925 GMT) and the 500-mb analysis (1200 GMT), this splitting coincides with the upper trough position. The same inference can be made from the neph for the next day (Fig. 11).

On 20 April, the satellite did not acquire pictures over the main closed system which is still reasonably vertical. Based on the conventional data, the $500-\mathrm{mb}$ center north of Lake Superior is west of the surface center over James Bay. The cloud mass indicated as a "possible vortex" over Vermont is the now weakened remanants of the center which was in the trough over Minnesota 24 hours earlier. Heavy cloud cover can be seen near the $500-\mathrm{mb}$ ridge line which runs northsouth along $65 \mathrm{~W}$. It results from vorticity advection associated with the $500-\mathrm{mb}$ trough from James Bay to Pennsylvania. This trough has moved eastward at 

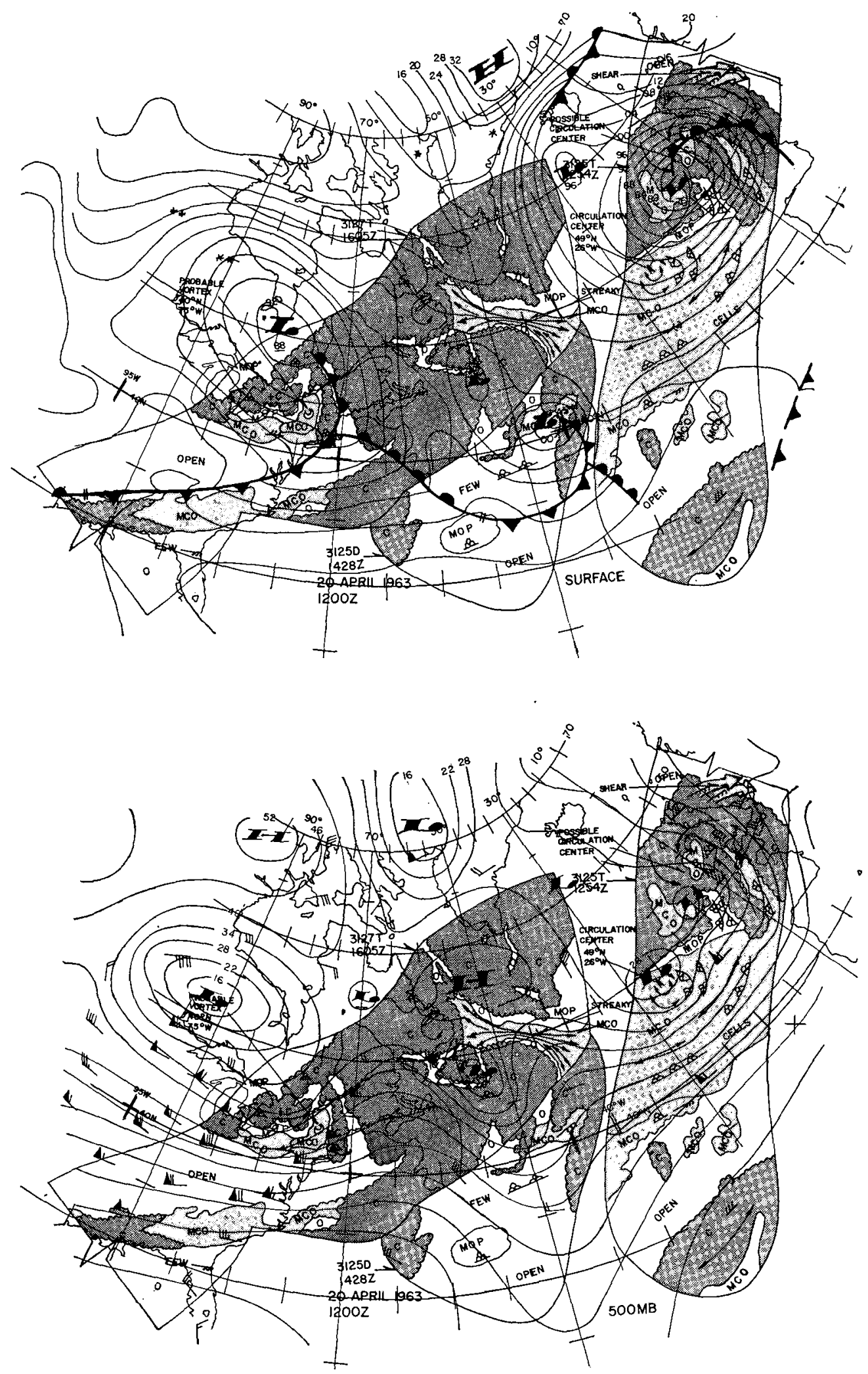

FIG. 11. Neph mosaic and conventional analysis for 1200 GMT, 20 April 1963. 
nearly 50 knots; thus the four hour difference between neph and analysis time would account for the area of heavy covered cloudiness $(+\mathrm{C})$ appearing to extend across the $500-\mathrm{mb}$ ridge line over New Brunswick. The distinction between the heavy covered areas and the perhaps less significant areas of broken and covered cloudiness to the east, as depicted on the neph, was clearly visible in the TIROS pictures upon which the neph was based. The surface analysis shown in Fig. 11 is also four hours earlier than the neph but, even so, the cloud pattern suggests the northern portion of the cold front and the point of occlusion probably should be
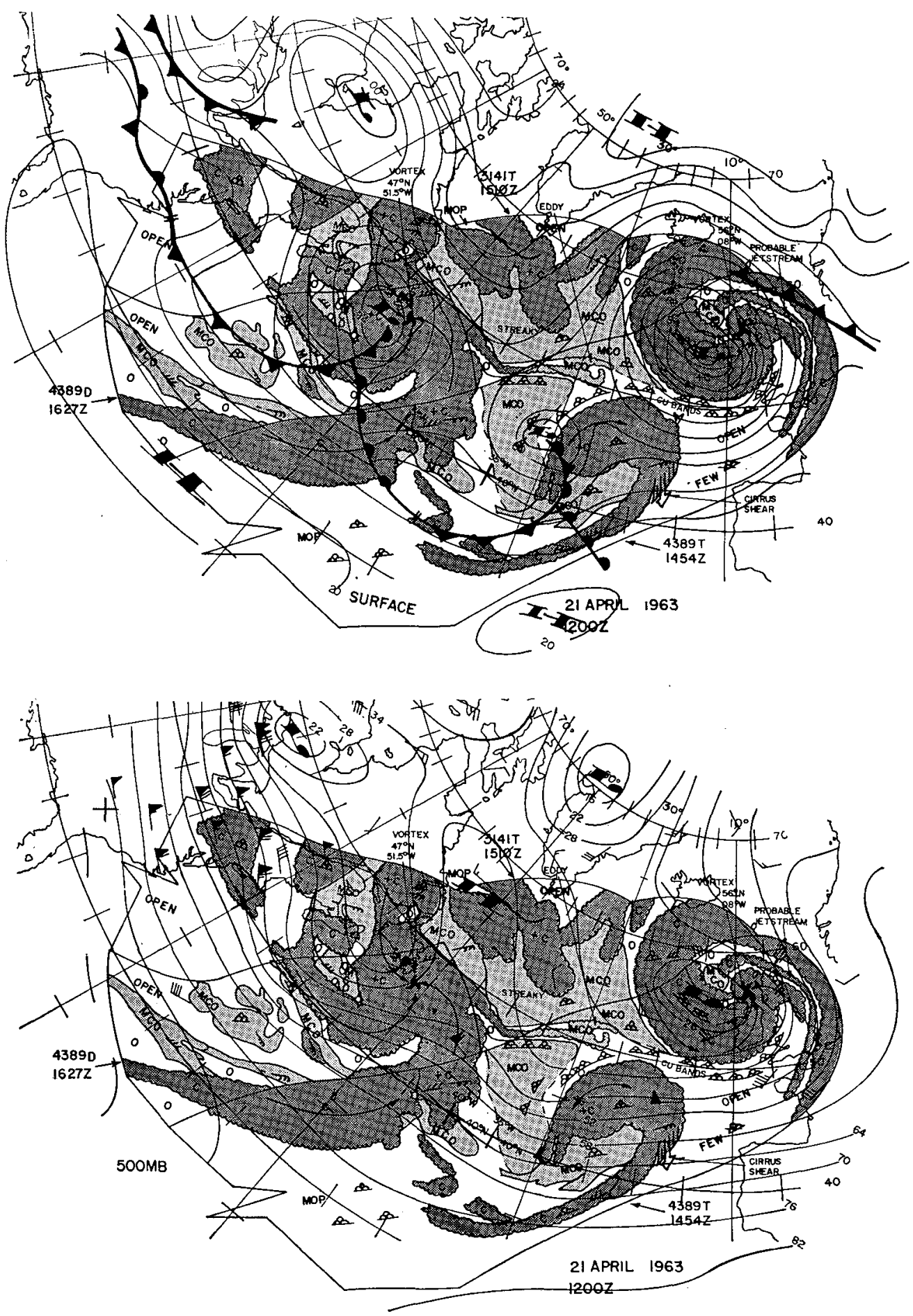

Fic. 12. Neph mosaic and conventional analysis for 1200 GMT, 21 April 1963. 
positioned farther east or northeast by at least 5 degrees of longitude.

The cloudiness over eastern Canada merges with that associated with another storm to the east which has moved from eastern Quebec and is over Newfoundland by 1200 GMT 20 April. The cloud outline to the southeast of Newfoundland suggests a well defined trough extending south of the center. Farther to the southeast, near $44 \mathrm{~N}, 44 \mathrm{~W}$, another cloudy area is associated with the southern extension of this trough and will be discussed in a later paragraph.

During the next 24 hours, the cloud pattern southeast of Newfoundland becomes more organized and a vortex is indicated at $47 \mathrm{~N}, 51.5 \mathrm{~W}$ (Fig. 12). The clear area south of New Brunswick, coupled with the $500-\mathrm{mb}$ wind shifts at Nantucket and Caribou, indicate that a trough has passed in the last 24 hours. Clear skies now indicate a ridge. The trough which passed Nantucket, moved into the area west of the system which at 1200 GMT 20 April, was centered over Newfoundland. The neph for 21 April (Fig. 12) indicates the increased organization of the pattern and a probable increased intensity of the storm.

The cloud pattern at 1600 GMT would suggest a more fully occluded stage than that indicated on the surface analysis with the cold front extending along the cloud band from $40 \mathrm{~N}, 45 \mathrm{~W}$ to $35 \mathrm{~N}, 50 \mathrm{~W}$. This would place the front nearly 10 degrees farther east at $40 \mathrm{~N}$. The surface data would easily allow the front to be reanalyzed to better fit the cloud pattern. With the cloud organization shown, one might reasonably forecast the closing off of a $500-\mathrm{mb}$ center near $50 \mathrm{~N}$ in the very near future and the possible further occluding of the surface storm.

Small system in the mid-Atlantic. In Fig. 9 a small "blob" of heavy cloud is apparent in the mid-Atlantic near $40 \mathrm{~N}$, 50W. An analyst seeing this type of cloud pattern should suspect a wave in the midtropospheric contour field and a possible development of a surface system nearly under the cloud form (Widger, 1964). An upper level trough is apparent in the $500-\mathrm{mb}$ contour pattern along $57 \mathrm{~W}$ between 36 and $41 \mathrm{~N}$. The cloud pattern suggests that this short wave trough is not the same trough that appears to begin at $44 \mathrm{~N}$ and runs between Nova Scotia and Newfoundland into the low pressure area over eastern Quebec. By 1200 GMT 20 April (Fig. 11), the trough which was more or less along $63 \mathrm{~W}$ on 19 April has moved to $52 \mathrm{~W}$ and has been analyzed as extending southward to $30 \mathrm{~N}$ along $50 \mathrm{~W}$. The neph shows a comma shaped cloud mass along $45 \mathrm{~W}$ between 40 and $45 \mathrm{~N}$. This cloud mass indicates that the short wave along $57 \mathrm{~W}, 24$ hours before, has moved to $47 \mathrm{~W}$. Using this information an analyst might tend to draw less of a sharpening and southward extension of the westernmost trough along $50 \mathrm{~W}$ and instead place a short wave trough near $40 \mathrm{~N}, 45 \mathrm{~W}$. This would lead to a better indication of the events to take place in the eastern Atlantic during the next 24 hours.
The surface analysis near $40 \mathrm{~N}, 45 \mathrm{~W}$ shows a frontal system with a small occlusion. Even taking the $2 \mathrm{hr}$ 30 min time difference into account, the cloud pattern would still indicate that this low level system should be at least 5 degrees farther east and 4 to 5 degrees farther north. The surface map for 1200 GMT shows no data that would prohibit such a reanalysis. It seems reasonable to assume that the system is more likely to be where the cloud pattern indicates than where the NMC analyst placed it based on sparse conventional data alone.

By 1450 GMT 21 April (Fig. 12), the cloud pattern near $45 \mathrm{~N}, 27 \mathrm{~W}$ has the comma shape which Rogers (Widger, 1964) has shown to be associated with the combination of vorticity and thermal advection downwind of an open wave pattern at the $500-\mathrm{mb}$ level. Recognition of this pattern often allows $500-\mathrm{mb}$ contour patterns to be estimated with some precision over data sparse areas. In this case the trough is verified by the data from ocean ship "Delta" $(45 \mathrm{~N}, 41 \mathrm{~W})$ and from Lages $(38 \mathrm{~N}, 27 \mathrm{~W})$. The associated surface system should be centered near $45 \mathrm{~N}, 26 \mathrm{~W}$, with the cold front along the cloud band (Fig. 12). No data would be violated if the system were moved to fit the cloud pattern. Thus the cloud pattern coupled with the conventional data leads to a better analysis. In this case, the surface system would be moved some 250 or more miles closer to the coast of Spain and would materially affect the forecast for that area in the subsequent period.

The double vortex pattern over the central North Atlantic. A double vortex in the North Atlantic can be clearly seen in Fig. 9. The cloud system centered near $50 \mathrm{~N}$, $39 \mathrm{~W}$ appears to be closed at $500 \mathrm{mb}$ and shows a heavy band to the northwest of the center. This pattern usually occurs with cyclonic curvature of the $500-\mathrm{mb}$ contour field in the area of the heavy cloudiness (Personal communication from Mr. Vincent Oliver, USWB, May 1964.) This example suggests how an analyst might construct the contours near such a cloud pattern if little other data were available.

This double system weakens (Fig. 11) while the upper level flow favors intensification of the new closed center that developed from the trough which was near $44 \mathrm{~N}, 26 \mathrm{~W}$ and is now near $52 \mathrm{~N}, 12 \mathrm{~W}$. The new system had considerable organization by 21 April (Fig. 12) and now looks like a fully occluded vortex.

\section{Some deductions from these cases}

These cases have pointed out some of the advantages to be gained from the compositing of nephs and the superposing of conventional analyses. The resultant maps suggested several areas where an improved synoptic analysis could have been made. Time-tested techniques such as continuity, when used to supplement the integrated data, also aided in the positioning and interpretation of major synoptic patterns. Over 
data sparse areas, as in the April case example, these procedures can lead to better analyses and so to improved forecasts.

\section{Summary}

The operational procedures suggested above have been cited for purposes of illustration. At this stage in the development of the operational use of satellite data, it is neither possible nor perhaps desirable to prescribe specific procedures for all kinds of situations. The forecaster must ultimately decide what is best in each individual case. For this purpose, the following principles are suggested as general guidelines:

1) Satellite data become most useful when fully integrated with meteorological information from all other sources. Because of this, integrating satellite cloud data with other synoptic charts is very desirable. In this manner, as much pertinent data as possible is assembled on one chart.

2) However, at field weather stations, transpose only such data as have the prospect of being useful. "Time is a precious commodity for the operational forecaster, especially during periods of critical weather. He must, therefore, limit his procedures to those that hold most promise of being productive" (Hanson, 1963).

3) The nephanalyses can provide the forecaster with data otherwise unobtainable. Compositing the nephs and their integration with conventional synoptic analyses (or simplified versions thereof) aids the operational interpretation and application of the satellite data.

4) As in all meteorological analysis, immediate past as well as current nephanalyses should be examined and considered. While the forecaster should not become a slave of continuity, neither should a reasonable continuity of the movement and development of systems be abandoned without reasons for so doing being noted in the data.

In some areas of the world, no conventional data exist for hundreds of miles. In these areas, it is suggested that characteristic cloud patterns (Widger,
1964; Widger et al., 1964), can often be associated with such features as typical frontal patterns, fields of vertical motion, or areas of significant vorticity advection, and that even remote conventional observations can often be logically tied into an analysis once the basis for the analysis is established by the satellite data. Such an improved analysis is a first and necessary step to a better forecast.

Acknowledgments. The author is grateful for the many helpful suggestions and the encouragement provided by Dr. William K. Widger, Jr. and Mr. C. William C. Rogers during the preparation of this report.

\section{REFERENCES}

Boucher, R. J., C. J. Bowley, E. S. Merritt, C. W. C. Rogers, P. E. Sherr and W. K. Widger, Jr., 1963: Synoptic interpretations of cloud vortex patterns as observed by meteorological satellite. Final Report, Contract No. Cwb-10630, ARACON Geophysics Company (available DDC, No. 609493, or OTS).

Brodrick, H. J., Jr., 1964: TIROS cloud pattern morphology of some mid-latitude weather systems. Meteorological Satellite Laboratory Report No. 24, National Weather Satellite Center, U. S. Weather Bureau.

Hanson, D. M., 1963: The use of meteorological satellite data in analysis and forecasting. Tech. Note No. 13, Office of Forecast Development, U. S. Weather Bureau (available from National Weather Satellite Center).

Miller, F. R., 1963: An application of TIROS cloud observations in sparse data regions. Mon: Wea. Rev., 91, 433-451.

Oliver, V. J., 1962: Comparison of a satellite nephanalysis for a family of pacific frontal storms. Final Report on the TIROS I Meteorological Satellite System, Tech. Report No. R-131, National Aeronautics and Space Administration.

Stampfl, R. A., and W. G. Stroud, 1963: The automatic picture transmission (APT) TV camera system for meteorological satellites. Tech. Note No. D-1915, National Aeronautics and Space Administration.

Widger, W. K., Jr., 1964: A synthesis of interpretations of extratropical vortex patterns as seen by TIROS. Mon. Wea. Rev., 22, 263-282.

-_, 1966: Meteorological Salellites. New York, Holt, Rinehart and Winston, $280 \mathrm{pp}$.

—- Paul E. Sherr and C. W. C. Rogers, 1964: Practical interpretation of meteorological satellite data. Final Report, Contract No. AF 19(628)-2471 (available from DDC, AD 436810 , or OTS). (Reprinted as AWSTR 185.) 